\title{
Hsa-miR-34c suppresses growth and invasion of human laryngeal carcinoma cells via targeting c-Met
}

\author{
KE-MIN CAI, XUE-LI BAO, XU-HUI KONG, WU JINAG, MING-RONG MAO, \\ JIU-SHENG CHU, YONG-JIU HUANG and XIAO-JUN ZHAO \\ Department of Otorhinolaryngology Head and Neck Surgery, Taizhou People's Hospital, \\ Taizhou 225300, Jiangsu, P.R. China
}

Received November 2, 2009; Accepted December 7, 2009

DOI: 10.3892/ijmm_00000378

\begin{abstract}
MicroRNAs (miRNAs) are small noncoding RNA molecules that negatively modulate gene expression at the post-transcriptional level. A growing number of studies has shown that more and more miRNAs are aberrantly expressed and involved in the pathogenesis of several types of cancers. Here, we report that the down-regulated hsa-miR-34c was also involved in oncogenesis of laryngeal carcinoma. Our studies indicated that hsa-miR-34c functioned as a tumor suppressor which inhibited growth and invasion of human laryngeal carcinoma cells. Furthermore, in our study, an inverse relationship between the expression of hsa-miR-34c and c-Met was identified in 10 paired fresh samples from tumor tissues and adjacent normal tissues. Infection of hsamiR-34c mediated by lentivirus suppressed the expression of c-Met directly. In addition, introduction of c-Met cDNA lacking 3'-UTR largely abrogated hsa-miR-34c-induced cell growth and invasion inhibition. These findings suggest aberrantly down-regulated hsa-miR-34c is a critical factor that contributes to malignancy in human laryngeal carcinoma by a mechanism involving targeting of c-Met.
\end{abstract}

\section{Introduction}

MicroRNAs (miRNAs) are a group of 22-nucleotide endogenous noncoding RNA, which regulate gene expression at the post-transcriptional level. miRNAs are initially transcribed as primary RNA transcripts (termed pri-miRNAs). These primary transcripts are then processed into an $~ 70$-nt stem-loop hairpin precursor (called pre-miRNAs) in the nucleus by an RNase III enzyme (Drosha). Then pre-miRNAs are transported to the cytoplasm where they are processed to generate 22 -nt mature miRNAs by Dicer. Finally, the mature single-stranded miRNA

Correspondence to: Dr Kemin Cai or Dr Xue-Li Bao, Department of Otorhino-laryngology Head and Neck Surgery, Taizhou People's Hospital, Taizhou 225300, Jiangsu, P.R. China

E-mail: njmucaikemin@126.com

E-mail: njmubaoxueli@126.com

Key words: microRNA, hsa-miR-34c, apoptosis, proliferation, invasion, laryngeal carcinoma is incorporated into the RNA-induced silencing complex (RISC), which binds mRNA with partial complementarity and leads to translational repression in mammals (1). In recent years, abnormal expressions of many miRNAs have been found in several tumor types, such as breast carcinoma, glioblastoma, lung cancer, malignant lymphomas and B cell lymphomas (2).

Laryngeal carcinoma is one of the most common head and neck cancers. Although early-stage laryngeal cancer is often curable via surgery or radiotherapy, the advanced disease is lethal for the majority of patients. In the last two decades, the outcome has not improved greatly despite therapeutic advances. In order to further improve the prognosis of the disease, it is necessary to better understand the mechanisms of carcinogenesis. Despite great efforts made in recent years, the molecular mechanism involved in the initiation and progression of laryngeal carcinoma remains largely unclear (3).

In human laryngeal carcinoma cells, Liu et al reported that miR-21 was overexpressed in human laryngeal carcinoma cells, and acted as an antiapoptotic factor (4). In this study, we analyzed the effects of hsa-miR-34c on human laryngeal carcinoma Hep-2 cells, which are sharply down-regulated in human laryngeal carcinoma when compared with its paired normal tissues. Lentivirus-mediated hsa-miR-34c (lenti-miR$34 \mathrm{c})$ suppressed growth and invasion of Hep-2 cells. c-Met, which was identified as an miR-34c target gene, may be responsible for the lenti-miR-34c-induced growth and invasion inhibition. Our data showed hsa-miR-34c functions as tumor suppressor in human laryngeal carcinoma.

\section{Materials and methods}

Cell lines and culture conditions. Hep-2, human laryngeal carcinoma cell line, was purchased from the Chinese Academy of Sciences Cell Bank. Hep-2 was maintained in a $37^{\circ} \mathrm{C}, 5 \% \mathrm{CO}_{2}$ incubator in DMEM supplemented with $10 \%$ fetal bovine serum (FBS), and routinely passaged at 2- to 3-day intervals. Experiments were divided into three groups as blank control group (blank), miRNA scrambled group (scramble), has-miR-34c group (lenti-miR-34c).

RNA isolation, RT-PCR and real-time quantification of miRNAs by stem-loop RT-PCR. Laryngeal carcinoma 
specimens were collected from the Taizhou First People's Hospital after informed consent from adult patients diagnosed with laryngeal carcinoma requiring surgical resection. The paired fresh samples from tumor tissues and adjacent normal tissues were freshly resected during surgery and immediately frozen in liquid nitrogen for subsequent total RNA extraction. The primers used in RT-PCR are as follows: c-Met, 5'-TGG GAA TCT GCC TGC GAA-3' and 5'-CCA GAG GAC GAC GCC AAA-3'; ß-actin, 5'-CCA ACC GCG AGA AGA TGA-3' and 5'-CCA GAG GCG TAC AGG GAT AG-3'. All primers and probes of hsa-miR-34c and RNU6B endogenous controls for TaqMan miRNA assays were purchased from Applied Biosystems. Real-time PCR was performed according to the manufacturer's instructions. The relative gene expression was calculated via a $2-\Delta \Delta \mathrm{Ct}$ method.

Lentiviral vector construction and infection. Expression vector mediated by lentivirus for hsa-miR-34c was constructed via technical support from Xiasi Bio (Nanjing). The full length of pre-hsa-miR-34c was annealed and inserted into plenti-LV vector (Invitrogen), and identified by restriction endonuclease digestion and nucleotide sequencing. The prehsa-miR-34c expression vectors (pGC-LV) and packaging vectors (pHelper 1.0 and 2.0) were cotransfected into 293FT cells with Lipofectamine 2000 (Invitrogen). After $48 \mathrm{~h}$, the culture supernatants were collected, concentrated and stocked at $-70^{\circ} \mathrm{C}$. Hep- 2 cells were infected with lentiviral vectors with a multiplicity of infection (MOI) at 10 in the presence of polybrene $(10 \mu \mathrm{g} / \mathrm{ml})$.

Western blot assay. To determine the levels of protein expression, cells were lysed by lysis buffer $\left(15 \mathrm{mM} \mathrm{MgCl}_{2}\right.$, $0.1 \%$ Triton X-100, $25 \mathrm{mM}$ MOPS, $137 \mathrm{mM} \mathrm{NaCl}, 15 \mathrm{mM}$ EGTA, $0.1 \mathrm{mM}$ sodium orthovanadate, $100 \mu \mathrm{M}$ phenylmethylsulfonyl fluoride and $20 \mu \mathrm{M}$ leupeptin, adjusted to $\mathrm{pH}$ 7.2). Equal amounts of proteins $(30 \mu \mathrm{g})$ were separated by $12 \%$ sodium dodecyl sulfate (SDS)-polyacrylamide gel electrophoresis using the discontinuous buffer system of Laemmli (Bio-Rad Laboratories, Richmond, CA). The separated proteins were transferred to PVDF membrane and subjected to immunoblot analysis with antibody c-Met (Santa Cruz Biotechnology). The reaction was visualized with enhanced chemiluminescence (Amersham Life Science, Arlington Heights, IL). GAPDH was taken as internal control.

Cell growth assay. Relative cell growth was determined using MTT assays. Briefly, the Hep-2 cells infected by lentimiR-34c were seeded at $10^{3}$ cells per well in 96-well plates, infected and analyzed by MTT assays (Sigma) at 24, 48, 72 and $96 \mathrm{~h}$ post-transfection. Cells were incubated with $100 \mu \mathrm{l}$ of culture media supplemented with $10 \mu 1$ of MTT solution for $2 \mathrm{~h}$ at $37^{\circ} \mathrm{C}$. The cell viability was determined at $570 \mathrm{~nm}$ absorbance. Each experiment was performed in triplicates. Cell growth inhibition rate formula is (AC-AT)/ACx $100 \%$ (AT, absorbance value of the experimental group; AC, absorbance value of the blank control group).

Soft agar colony assay. Anchorage-independent glioma cell growth was determined by Soft agar colony assay. Hep-2 cells infected with lenti-miR-34c and scramble were seeded in $0.35 \%$ agar in the middle agar with $0.5 \%$ agar plated at the bottom. After incubation in a $37^{\circ} \mathrm{C}, 5 \% \mathrm{CO}_{2}$ incubator for two weeks, colonies were photographed and counted under a microscopic field at $\mathrm{x} 10$ fields. Each assay was performed in triplicate on five independent occasions.

Invasion assay. Transwell membranes coated with Matrigel (BD Biosciences, San Jose, CA) were used for invasion assay of Hep-2 cells in vitro. Serum-free medium containing $5 \times 10^{4}$ infected cells was plated in the upper chamber. The media containing $20 \%$ FBS was added to the lower chamber as attractant. After incubating $24 \mathrm{~h}$, noninvading cells were removed from the top well with a cotton swab while the bottom cells were fixed with $75 \%$ alcohol, stained with $0.1 \%$ crystal violet, and photographed in three independent x 10 fields for each well. Five independent experiments were performed.

TUNEL assay. The apoptosis of cells was determined using the TUNEL assay. Hep-2 cells infected by lenti-miR-34c and scramble for $24 \mathrm{~h}$ were fixed by $4 \%$ paraformaldehyde and incubated with TUNEL reaction mixture. Apoptotic cells were observed under fluorescence microscope (Olympus, Tokyo, Japan).

Luciferase reporter assay. Construction of plasmids containing 3'UTR-c-Met (p3'UTR-c-Met) and 3'UTRmutation-c-Met (p3'UTR-mut-c-Met) were constructed via technical support from Xiasi Bio (Nanjing). 3'-UTR sequence of c-Met predicted by TargetScan to interact with hsa-miR$34 \mathrm{c}$ and a mutated sequence of the 3'-UTR sequence was inserted into pGL3 vectors (Promega). After infection of lenti-miR-34c for $24 \mathrm{~h}$, Hep-2 cells were transfected with p3'UTR-c-Met and p3'UTR-mut-c-Met plasmids. After $48 \mathrm{~h}$, Luciferase activity of Hep-2 cells was measured $96 \mathrm{~h}$ after infection using the Dual-Luciferase reporter assay system (Promega).

Statistical analysis. All tests were analyzed using SPSS Graduate Pack 11.0 statistical software (SPSS, Chicago, IL). Data including mean and \pm SE along with one-way ANOVA were used to determine significant differences. $\mathrm{P}<0.05$ was considered significant.

\section{Results}

Hsa-miR-34c was down-regulated in human laryngeal carcinoma. To investigate whether hsa-miR-34c was downregulated in human laryngeal carcinoma as reported by Liu et al (4), we performed the TaqMan-based real-time stem-loop RT-PCR analysis. As shown in Fig. 1, our data confirmed that hsa-miR-34c was strongly down-regulated in human laryngeal carcinoma versus paired normal laryngeal tissues. The normal laryngeal tissues showed remarkable expression of hsa-miR$34 \mathrm{c}$, while the level of hsa-miR-34c was very low in human laryngeal carcinoma tissues.

Hsa-miR-34c inhibited the growth of Hep-2 cells in vitro. The in vitro proliferation assay of infected Hep-2 cells was determined by MTT assay. To study the biological significance of elevated hsa-miR-34c levels in laryngeal carcinoma cells, 


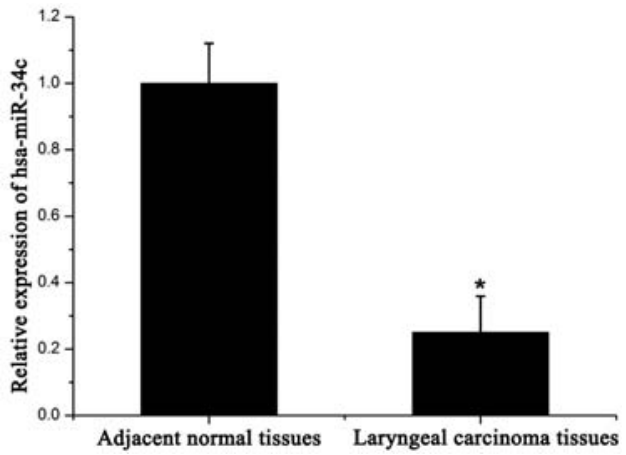

Figure 1. Expression of hsa-miR-34c in human laryngeal carcinoma. " $\mathrm{p}<0.05$ versus normal tissues.

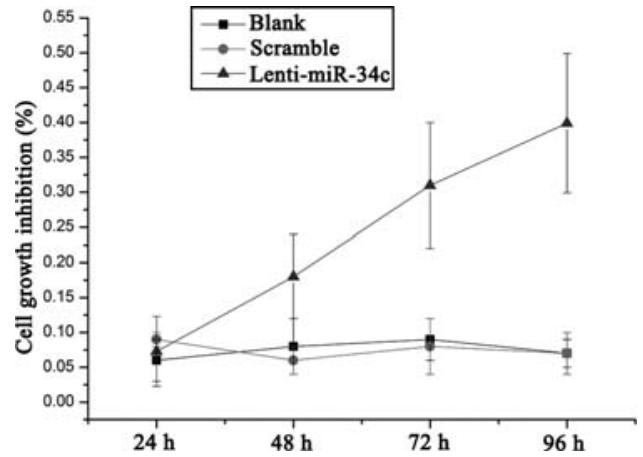

Figure 2. Effects of hsa-miR-34c on the proliferation of Hep-2 cells. The cell growth inhibition rates of Hep-2 after infection of lenti-miR-34c, scramble and blank controls at different time point.

A

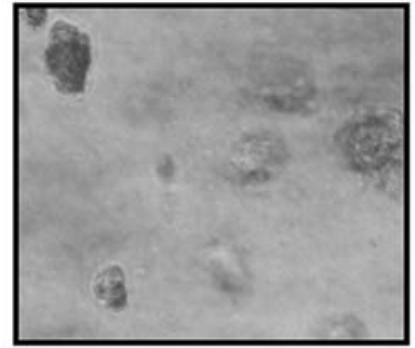

Blank

B

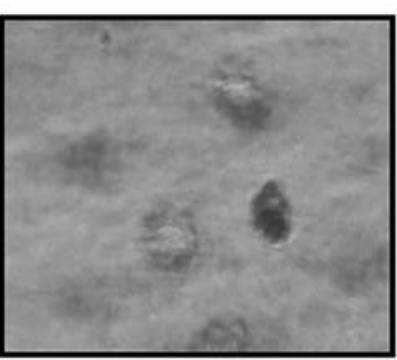

Scramble

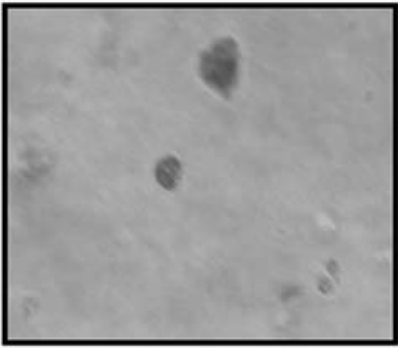

Lenti-miR-34c

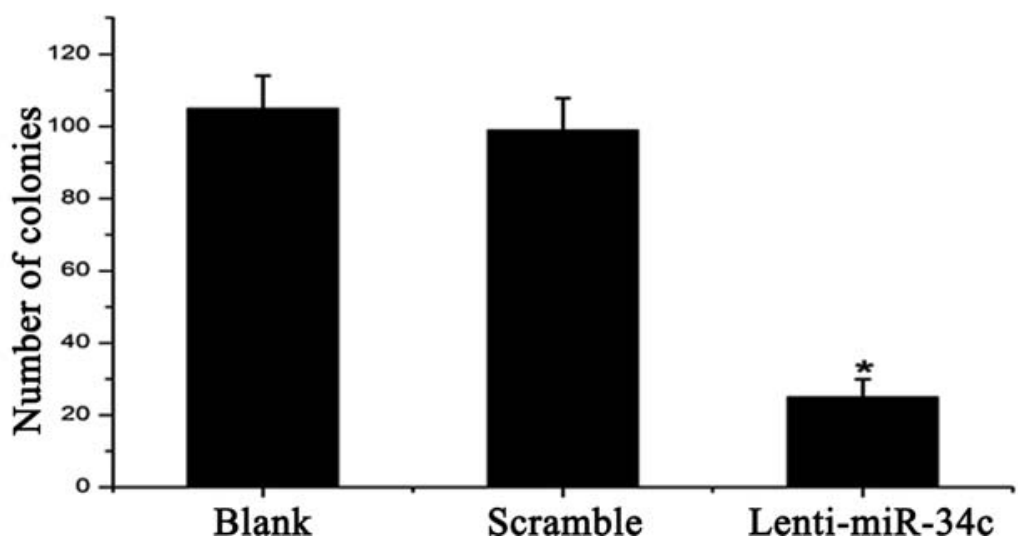

Figure 3. Hsa-miR-34c inhibited neoplastic transformation of Hep-2 cells. (A) Hep-2 cells were infected with lenti-miR-34c. Two weeks after infection, plates were fixed and photographed (Magnification, x100). (B) Columns, number of colonies from five independent experiments, each in triplicate; bars, \pm SE. Statistically significant differences from scramble and blank controls were observed; ${ }^{*} \mathrm{P}<0.05$ versus scramble and blank controls.

we first infected the laryngeal carcinoma cell line, Hep-2, with lenti-miR-34c. As shown in Fig. 2, infection of Hep-2 cells with lenti-miR-34c caused suppression of cellular proliferation at 72 and $96 \mathrm{~h}$ post-infection when compared with scramble and blank controls. Meanwhile, anchorage-independent cell growth was determined by soft agar analysis in our study. As shown in Fig. 3, the number of colonies from HEp-2 cells infected with lenti-miR-34c was significantly lower than that of scramble and blank controls. This implied that hsa-miR-34c suppresses cell growth significantly in human laryngeal carcinoma.
Hsa-miR-34c induced apoptosis of Hep-2 cells in vitro. To study the effect of hsa-miR-34c, we infected Hep-2 cells with lenti-miR-34c. After post-infection for $72 \mathrm{~h}$, its apoptosis was measured by TUNEL assay. As shown in Fig. 4, statistically significant more TUNEL-positive cells were found in lentimiR-34c infected cells than scramble and blank controls (Fig. 4). Thus, we concluded that hsa-miR-34c induces apoptosis in human laryngeal carcinoma.

Hsa-miR-34c suppressed the invasion of Hep-2 cells in vitro. To measure the tumor cell invasion ability after infection, the in vitro invasion assay was employed. We investigated 


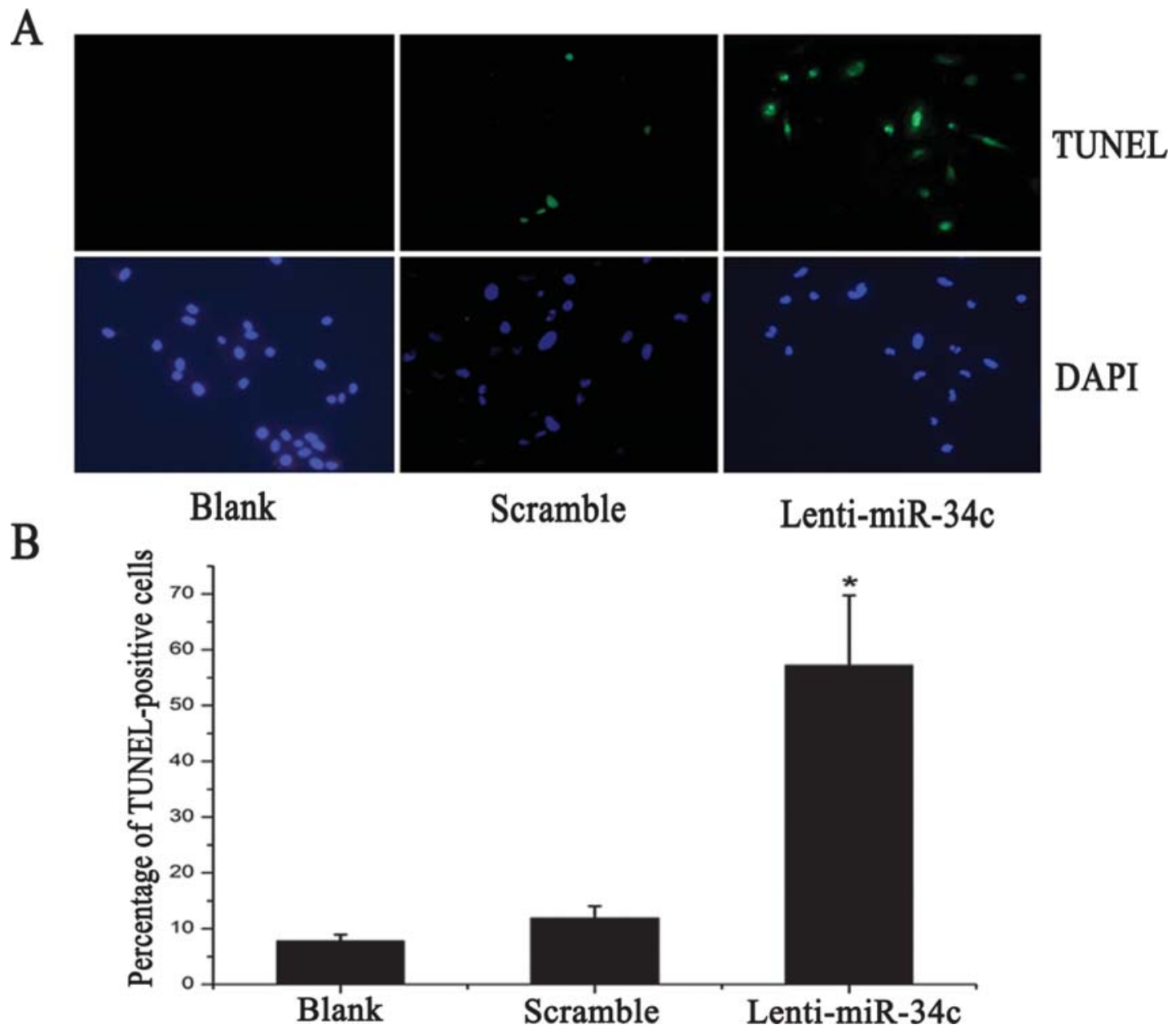

Figure 4. Effects of hsa-miR-34c on apoptosis of Hep-2 cells. After infection of hsa-miR-34c and controls for 72 h, cells were fixed with $4 \%$ paraformaldehyde and incubated with TUNEL reaction mixture. (A) Apoptotic cells were observed and photographed under fluorescence microscope. (B) Columns, number of TUNEL-positive cells from five independent experiments, each in triplicate; bars, \pm SE. Statistically significant differences from scramble and blank controls were observed. ${ }^{*} \mathrm{p}<0.05$ versus scramble and blank controls.

whether hsa-miR-34c affects invasion of Hep-2 cells using transwells. In this test, only invasive cells had the potential to migrate through the pores in the membrane. As shown in Fig. 5, cells infected with lenti-miR-34c showed an average slightly $>60 \%$ defective in migrating through a basement membrane compared with scramble and blank controls. Our results strongly indicated that hsa-miR-34c was an important participant in the reduction of invasion potential of laryngeal carcinoma cells.

c-Met is down-regulated in laryngeal carcinoma and is a putative target of hsa-miR-34c. microRNAs regulate a variety of cellular activities via regulating the expression of multiple target genes. To understand the mechanism of miR-34c on the motility of laryngeal carcinoma cells, we next identified the target genes that may participate in this process. As hsa-miR$34 \mathrm{c}$ has been validated as being down-regulated in laryngeal carcinoma, its target genes should be correspondingly upregulated in laryngeal carcinoma. It is well known that miRNAs usually bind the 3'-untranslated region (UTR) of the target mRNA with incomplete complementarity. Bioinformatics methods (PicTar and TargetScan) were used for target predication. C-MET, which is up-regulated in ten paired laryngeal carcinoma tissues compared with the adjacent normal tissues was predicted to be a direct target gene of miR-34c and confirmed by Luciferase report assay (Fig. 6) and Western blotting (Fig. 7).

Introduction of c-Met cDNA lacking 3'-UTR reduces cell growth inhibition induced by hsa-miR-34c. It has been documented that miRNAs induced translational inhibition of their target genes through base-pairing interactions in the mRNA 3'-UTR. Because miR-34c down-regulates c-Met through binding to 3'-UTR of c-Met mRNA, ectopic expression of c-Met by infection of the cDNA mediated by lentivirus (lenti-c-Met) that only contains the coding region of c-Met should escape the regulation by miR-34c and thus attenuate or decrease miR-34c induced growth inhibition. To this end, infection of lenti-miR-34c-infected Hep-2 cells with lenti-c-Met lacking 3'-UTR can rescue the growth inhibition induced by lenti-miR-34c. These results further indicate that c-Met is a major target of miR-34c and largely mediates miR-34c-induced growth and invasion inhibition in human laryngeal carcinoma.

\section{Discussion}

MicroRNAs (miRNAs) are a class of naturally occurring small non-coding RNA of $\sim 21-23$ nucleotides that regulate 


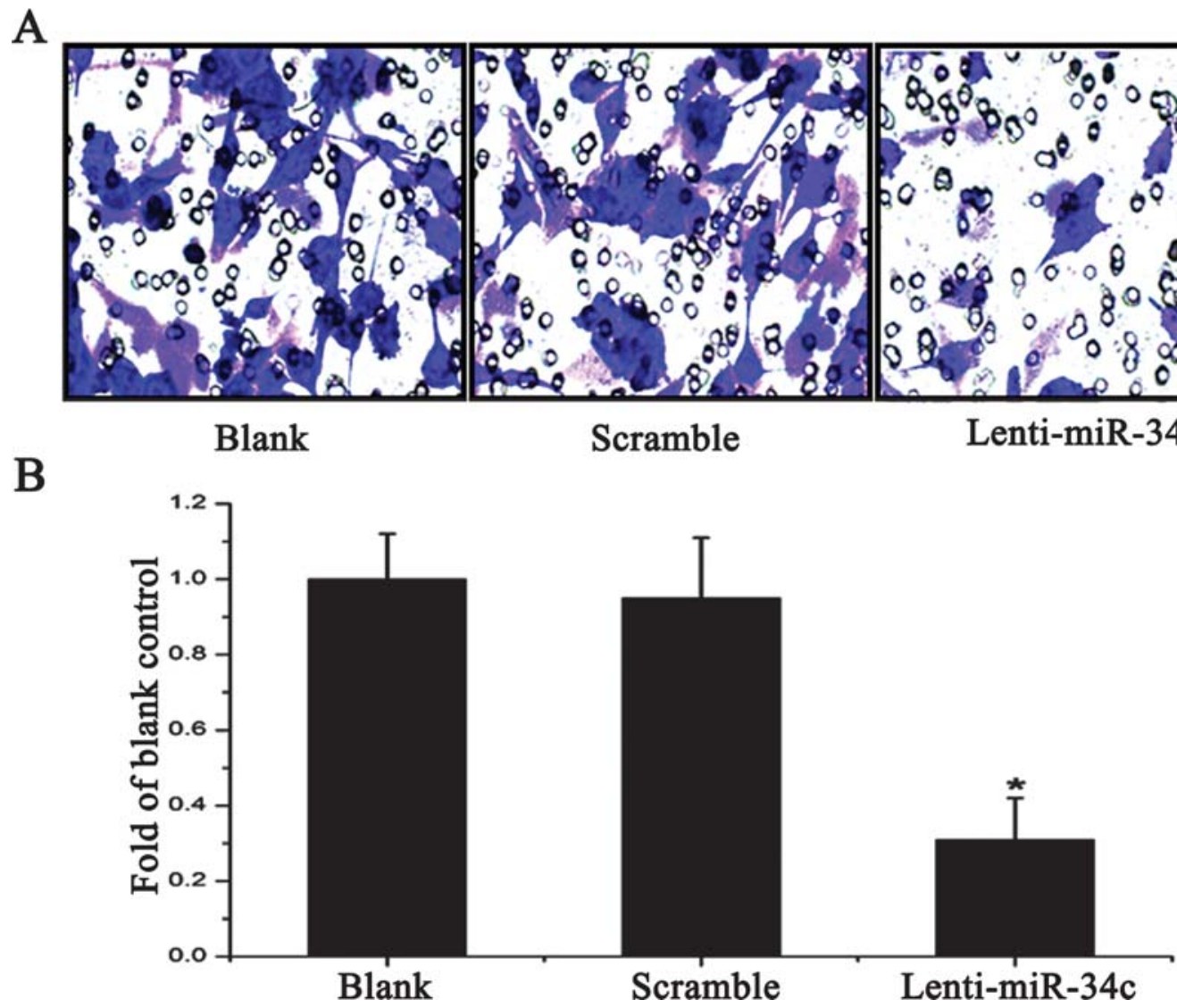

Figure 5. Effects of hsa-miR-34c on the invasion of Hep-2 cells. (A) Hep-2 cells infected with lenti-miR-34c scramble and blank controls for 72 h were plated on Matrigel-coated membranes in the upper chamber of transwells. Media containing $20 \%$ FCS was used as attractant. After 24 h, cells penetrating the membrane were fixed and $0.1 \%$ crystal violet stained as described in Materials and methods. (B) Hep-2 cells penetrating the membrane were represented as the fold over blank controls \pm SE for five wells. ${ }^{*} \mathrm{p}<0.05$ versus scramble and blank controls.

A

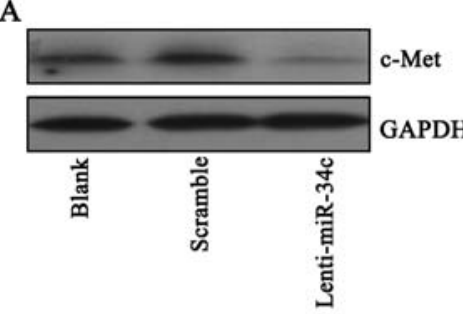

C

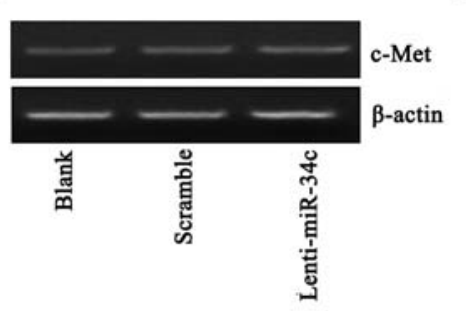

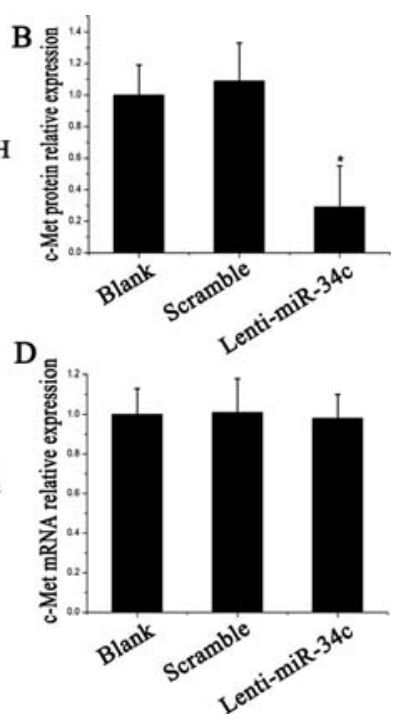

Figure 6. Regulation of c-Met expression by hsa-miR-34c. After infection for $72 \mathrm{~h}$, lenti-miR-34c mimics suppressed c-Met expression at protein levels (A). While, lenti-miR-34c did not affect the mRNA levels of c-Met (C). ${ }^{*} \mathrm{p}<0.05$ versus scramble and blank controls.

gene expression at the post-transcriptional level by degrading or repressing target mRNAs (5). To date, a growing number of studies has shown that more and more miRNAs are aberrantly expressed and involved in the pathogenesis of several types of cancers (6). In addition, microRNAs were found to regulate the expression of cancer-related genes (7-10). In the current study, we reported hsa-miR-34c was downregulated in human laryngeal carcinoma. Then, we further showed that infection of Hep-2 cells with lenti-miR-34c significantly induced growth and invasion inhibition. Furthermore, we found that c-Met was a putative target of hsa-miR-34c. Introduction of c-Met cDNA lacking 3'-UTR reduced cell growth and invasion inhibition was induced by hsa-miR-34c. These data suggest that hsa-miR-34c is a growth inhibitor in human laryngeal carcinoma.

The acquisition of abnormal growth rates is generally considered to be one of the in vitro properties associated with the malignancy of cells (11). Recent advances showed that some microRNA, such as hsa-miR-181 and hsa-miR-138 may function as the growth inhibitor of tumor cells $(12,13)$. The balance between proliferation and apoptosis is often disturbed and accounts greatly for tumorigenesis in cancers (14). In the present study, our results showed that infection of lenti-miR-34c induced apoptosis and proliferation of Hep-2 cells. Anchorage-independent growth is an important malignant property of tumor cells (15). To evaluate whether hsa-miR-34c affects anchorage-independent growth of laryngeal carcinoma cells, Hep-2 cells were infected with lenti-miR-34c. Our data showed hsa-miR-34c led to cell soft agar colony growth inhibition in Hep- 2 cells. The generation of invasiveness in 
A

Potential target regiong of c-Met :
Hsa-miR-34c :

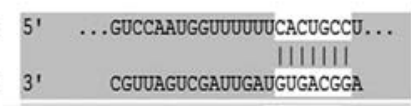

Potential target regiong of c-Met :

Hsa-miR-34c :

B

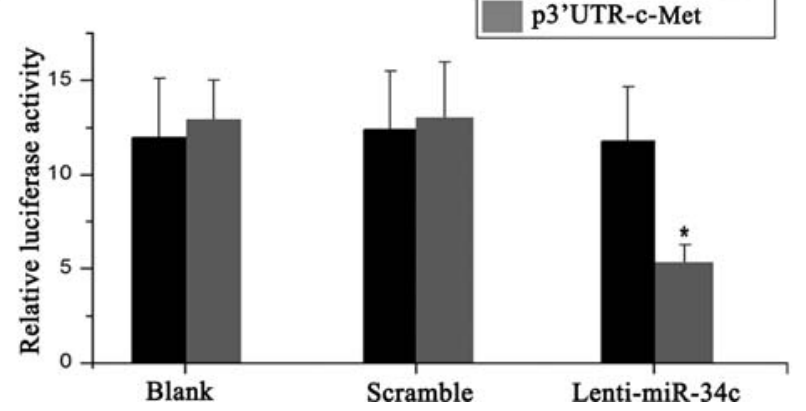

Figure 7. (A) Bioinformatics analysis of predicted interactions of hsa-miR-34c with their binding sequences in the 3'-UTR of c-Met (TargetScan). (B) Effect of hsa-miR-34c on their predicted sequences in the 3'-UTR of c-Met by luciferase reporter assay. After infection of lenti-miR-34c for $24 \mathrm{~h}$, both scramble and blank controls, Hep-2 cells were transfected with p3'UTR-cMet and p3'UTR-mut-c-Met plasmids. After 48 h, luciferase activity Hep-2 cells were measured using the Dual-luciferase reporter assay system. * $\mathrm{p}<0.05$ versus p3'UTR-mut-c-Met transfected cells.

A

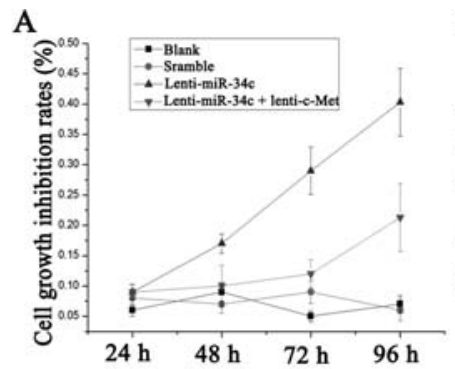

$\mathrm{C}$
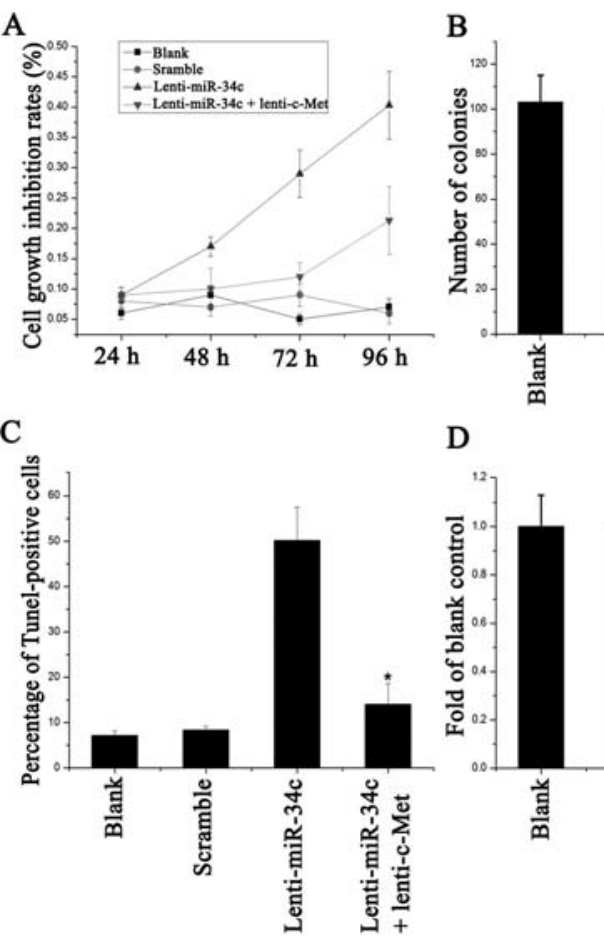

D

Figure 8. Hsa-miR-34c suppressed growth and invasion of Hep-2 cell via directly targeting c-Met. Introduction of lenti-c-Met cDNA lacking 3'-UTR promoted cell proliferation (A), colony formation (B) and invasion (D), which were suppressed by lenti-miR-34c. In addition, lenti-c-Met also reduced cell apoptosis induced by lenti-miR-34c (C). ${ }^{*} \mathrm{p}<0.05$ versus lentimiR-34c infected cells.

transformed cells represents an essential step of tumor progression (16). In our study, the invasion ability of Hep-2 cells infected with lenti-miR-34c was significantly suppressed.
These data suggest that hsa-miR-34c may function as tumor suppressor in human laryngeal carcinoma.

c-Met, also known as MET or hepatocyte growth factor receptor (HGFR), is a proto-oncogene that encodes the protein c-Met (17). Aberrantly active c-Met can trigger tumor growth. Previous studies have reported that c-Met is an oncogene that is up-regulated in human laryngeal carcinoma tissues $(19,20)$. We also found that c-Met was overexpressed in human laryngeal carcinoma tissues when compared with its adjacent normal tissues and negatively correlated with the levels of hsa-miR-34c (data not published). The bioinformatics analysis revealed that the evolutionary conserved binding sites on c-Met, which can be recognized by hsa-miR-34c, are all located in their 3'UTRs. Thus, we investigated whether hsamiR-34c suppressed cell growth and invasion via targeting c-Met. We found that hsa-miR-34c significantly downregulated c-Met protein levels, but not its mRNA levels. Luciferase reporter assays suggested that c-Met was one of the functional downstream targets of hsa-miR-34c in Hep2 cells. All the above suggest that c-Met is a potential functional target of hsa-miR-34c cells. Moreover, introduction of c-Met cDNA lacking 3'-UTR reduces cell growth and invasion inhibition induced by hsa-miR-34c. Furthermore, it can be concluded that hsa-miR-34c suppresses growth and invasion of laryngeal carcinoma cells via targeting c-Met.

In conclusion, hsa-miR-34c is down-regulated in laryngeal carcinoma tissues compared to their adjacent normal tissues. Overexpression of hsa-miR-34c suppressed cell growth and invasion of Hep-2 cells, possibly through inhibition of c-Met. These data suggest that the relationship of hsa-miR-34c and its target gene-c-Met may help us understand the molecular mechanism of the tumorigenesis of laryngeal carcinoma. Furthermore, the results in our study enhance our understanding of the potential function of miRNAs in laryngeal carcinoma. Hsa-miR-34c may function as a tumor suppressor in biotherapy in the future.

\section{Acknowledgements}

We are grateful to Xiasi Bio for technical support and the kind gift of lenti-c-Met. This work was supported by the Natural Foundation of Nantong Medical College.

\section{References}

1. Lai EC: microRNAs: runts of the genome assert themselves. Curr Biol 13: R925-R936, 2003.

2. Nelson KM and Weiss GJ: MicroRNAs and cancer: past, present, and potential future. Mol Cancer Ther 7: 3655-3660, 2008.

3. Marioni G, Marchese-Ragona R, Cartei G, Marchese F and Staffieri A: Current opinion in diagnosis and treatment of laryngeal carcinoma. Cancer Treat Rev 32: 504-515, 2006.

4. Liu M, Wu H, Liu T, Li Y, Wang F, Wan H, Li X and Tang H: Regulation of the cell cycle gene, BTG2, by miR-21 in human laryngeal carcinoma. Cell Res 19: 828-837, 2009.

5. Hernando E: microRNAs and cancer: role in tumorigenesis, patient classification and therapy. Clin Transl Oncol 9: 155-160, 2007.

6. Schickel R, Boyerinas B, Park SM and Peter ME: MicroRNAs: key players in the immune system, differentiation, tumorigenesis and cell death. Oncogene 27: 5959-5974, 2008.

7. Xia H, Qi Y, Ng SS, Chen X, Chen S, Fang M, Li D, Zhao Y, Ge R, Li G, Chen Y, He ML, Kung HF, Lai L and Lin MC: MicroRNA-15b regulates cell cycle progression by targeting cyclins in glioma cells. Biochem Biophys Res Commun 380: 205-210, 2009. 
8. Asangani IA, Rasheed SA, Nikolova DA, Leupold JH, Colburn NH, Post S and Allgayer H: MicroRNA-21 (miR-21) post-transcriptionally downregulates tumor suppressor Pdcd4 and stimulates invasion, intravasation and metastasis in colorectal cancer. Oncogene 27: 2128-2136, 2008.

9. Zhang C, Kang C, You Y, Pu P, Yang W, Zhao P, Wang G, Zhang A, Jia Z, Han L and Jiang H: Co-suppression of miR$221 / 222$ cluster suppresses human glioma cell growth by targeting p27kip1 in vitro and in vivo. Int J Oncol 34: 1653$1660,2009$.

10. Liu X, Yu J, Jiang L, Wang A, Shi F, Ye H and Zhou X: MicroRNA-222 regulates cell invasion by targeting matrix metalloproteinase 1 (MMP1) and manganese superoxide dismutase 2 (SOD2) in tongue squamous cell carcinoma cell lines. Cancer Genomics Proteomics 6: 131-139, 2009.

11. Smith HS, Hackett AJ, Riggs JL, Mosesson MW, Walton JR and Stampfer MR: Properties of epithelial cells cultured from human carcinomas and nonmalignant tissues. J Supramol Struct 11: 147-166, 1979.

12. Shi L, Cheng Z, Zhang J, Li R, Zhao P, Fu Z and You Y: hsamir-181a and hsa-mir-181b function as tumor suppressors in human glioma cells. Brain Res 1236: 185-193, 2008.

13. Liu X, Jiang L, Wang A, Yu J, Shi F and Zhou X: MicroRNA-138 suppresses invasion and promotes apoptosis in head and neck squamous cell carcinoma cell lines. Cancer Lett 286: 217-222, 2009 .
14. Cotter TG: Apoptosis and cancer: the genesis of a research field. Nat Rev Cancer 9: 501-507, 2009

15. Ward JA, Huang L, Guo H, Ghatak S and Toole BP: Perturbation of hyaluronan interactions inhibits malignant properties of glioma cells. Am J Pathol 162: 1403-1409, 2003.

16. Behrens J, Mareel MM, Van Roy FM and Birchmeier W: Dissecting tumor cell invasion: epithelial cells acquire invasive properties after the loss of uvomorulin-mediated cell-cell adhesion. J Cell Biol 108: 2435-2447, 1989.

17. You WK and McDonald DM: The hepatocyte growth factor/c-Met signaling pathway as a therapeutic target to inhibit angiogenesis. BMB Rep 41: 833-839, 2008.

18. Maulik G, Shrikhande A, Kijima T, Ma PC, Morrison PT and Salgia R: Role of the hepatocyte growth factor receptor, c-Met, in oncogenesis and potential for therapeutic inhibition. Cytokine Growth Factor Rev 13: 41-59, 2002.

19. Sawatsubashi M, Sasatomi E, Mizokami H, Tokunaga O and Shin T: Expression of c-Met in laryngeal carcinoma. Virchows Arch 432: 331-335, 1998.

20. Yücel OT, Sungur A and Kaya S: c-met overexpression in supraglottic laryngeal squamous cell carcinoma and its relation to lymph node metastases. Otolaryngol Head Neck Surg 130: 698-703, 2004. 